\title{
Hypothermia Masks Most of the Effects of Rapid Cycling VNS on Rat Hippocampal Electrophysiology
}

Wouter Van Lysebettens ${ }^{\mathrm{a}}$, Kristl Vonck ${ }^{\mathrm{a}}$, Lars Emil Larsen ${ }^{\mathrm{a}}$, Mathieu Sprengers ${ }^{\mathrm{a}}$, Evelien

Carrette, Jean Delbeke ${ }^{\mathrm{a}}$, Wytse Wadman ${ }^{\mathrm{a}, \mathrm{b}}$, Paul Boon ${ }^{\mathrm{a}}$, Robrecht Raedt ${ }^{\mathrm{a}}$

(a) Laboratory for Clinical and Experimental Neurophysiology, Neurobiology and Neuropsychology (LCEN3), Ghent University, Belgium

(b) Swammerdam Institute of Life Sciences, University of Amsterdam, The Netherlands

Background. We have previously demonstrated that vagus nerve stimulation (VNS) modulates hippocampal dentate gyrus (DG) electrophysiology and induces hypothermia in freely moving rats.

Objective. This study evaluated whether hippocampal CA1 electrophysiology is similarly modulated by VNS and to what extent this modulation is associated with VNS-induced hypothermia.

Methods. In six freely moving rats, a first 4h session of rapid cycling VNS (7s on/ 18s off) was applied, while CA1 evoked potentials, EEG and core temperature were recorded. In a second $4 \mathrm{~h}$ session, external heating was applied during the $3^{\text {rd }}$ and $4^{\text {th }}$ hour of VNS to counteract VNS-induced hypothermia.

Results. VNS induced a decrease in the slope of the field excitatory post synaptic potential (fEPSP), an increase in the population spike (PS) amplitude and latency, a decrease in theta (4-12 $\mathrm{Hz})$ and gamma $(30-100 \mathrm{~Hz})$ band power and theta peak frequency. External heating normalized body temperature during VNS and abolished the effects on fEPSP slope, PS latency and gamma band power. The effects on theta band power and theta peak frequency were only partially abolished and the effect on PS amplitude was inverted from an increase to a decrease.

Conclusions. Rapid cycle VNS modulates CA1 electrophysiology in a similar fashion as found earlier for DG, suggesting a more wide-spread VNS-induced effect on hippocampal electrophysiology. Normalizing core temperature during VNS elucidated that VNS-induced 
hypothermia directly influences several electrophysiological parameters in CA1 but also masks a VNS-induced reduction in neuronal excitability.

\section{Introduction}

Vagus nerve stimulation (VNS) is an approved therapy for drug-resistant epilepsy but the mechanism of action underlying a seizure-suppressing effect is still unclear (1). The main vagal relay site of the brain is the nucleus tractus solitarius (NTS). The NTS has many projections to other autonomic brainstem nuclei but also higher brain regions such as the amygdala, thalamus, and hypothalamus. Although there are no direct connections between the NTS and the hippocampus we previously showed potent suppression of hippocampal seizures by VNS (2). An important relay structure mediating these seizure-suppressing effects is the locus coeruleus (LC), the most important source of noradrenaline for the hippocampus (2-4). To further elucidate the nature of the seizure-suppressing action of VNS, we previously investigated the electrophysiological effects in the hippocampus in freely moving rats. Spontaneous EEG showed a decrease in theta peak frequency from 7 to $5 \mathrm{~Hz}$ and a decrease in power of theta and gamma oscillations (5). Perforant path evoked potential recording in the dentate gyrus demonstrated that VNS resulted in a decreased efficacy of synaptic transmission between the perforant path and the dentate granule cells, evident as a decreased slope of the field excitatory postsynaptic potential EPSP (fEPSP) and an increased latency of dentate granule cell firing (i.e. population spike latency). Despite decreased synaptic efficiency more granule cells fired in response to perforant path stimulation resulting in an increased population spike (PS) amplitude which indicates increased intrinsic excitability of the granule cells (5). In contrast, similar evoked potential experiments in anesthetized rats demonstrated increased synaptic efficacy in CA3 and dentate gyrus but a reduction in neuronal excitability $(6,7)$.

In a recent study, we found that VNS induces hypothermia in freely moving rats (8). The VNS paradigm producing the largest hypothermia (i.e. rapid cycle VNS: $7 \mathrm{sec}$ ON/18 sec OFF) resulted in most profound electrophysiological changes in the hippocampus. This observation, together with previous evidence of profound effects of changes in body temperature on hippocampal electrophysiology, suggests that hypothermia could be an important confounder when investigating the effect of VNS on hippocampal electrophysiology. This confounding influence might also 
underlie the contradictory effects of VNS on hippocampal electrophysiology in anesthetized (temperature-controlled) versus awake rats.

In this study, we investigated the electrophysiological effects of VNS in the CA1 subregions of the hippocampus of freely moving rats and applied external heating to compare VNS effects with and without the confounding effects of hypothermia.

\section{Methods}

\section{Animals}

Six male Sprague Dawley rats (Harlan, The Netherlands) weighing 350g were kept under environmentally controlled conditions and treated according to the guidelines approved by the European Ethics Committee (decree 86/609/EEC). The study protocol was approved by the Animal Experimental Committee of Ghent University Hospital (ECD 16/06).

\section{Surgery}

Animals were implanted with a custom-made spiral cuff VNS electrode (extensively described previously(9)). Next, animals were placed in a stereotactic frame for placement of both scalp and depth electrodes. Quadripolar electrodes were used for recording and stimulation. For recording, four polyimide insulated stainless steel wires (Medwire New York, U.S.A) with a bare wire diameter of $70 \mu \mathrm{m}$ and $300 \mu \mathrm{m}$ inter-tip distance were used. For stimulation, four polyimide insulated stainless steel wires (California Fine Wires, California, U.S.A) with $110 \mu \mathrm{m}$ bare wire diameter and a $220 \mu \mathrm{m}$ inter-tip distance were used. Scalp electrodes consisted of a copper wire soldered to a stainless steel micro screw ( $0.8 \mathrm{~mm}$ diameter, Plastics one). Two small craniotomies were made at the following coordinates relative to bregma: $-3 \mathrm{~mm}$ anteroposterior, $+1.5 \mathrm{~mm}$ lateral for stimulation of the Shaffer collateral fibers, $-5 \mathrm{~mm}$ anteroposterior, $+3 \mathrm{~mm}$ lateral for recording in the CA1 area. Two scalp electrodes and two stainless steel anchor screws were implanted in the os frontale, 3 anchor screws in the os parietale and 3 in the os occipitale. Quadripolar electrodes were lowered in the brain parenchyma through a small cut in the exposed dura in the craniotomies. Starting at a depth of $1 \mathrm{~mm}$, rectangular biphasic pulses of $100 \mu \mathrm{A}, 100 \mu$ s pulse width (per phase) were administered at $0.1 \mathrm{~Hz}$ through the stimulation electrode while both recording and stimulation electrodes were slowly lowered until a characteristic fEPSP and PS were detected. To prevent loss 
of EPs after surgery, the recording electrode was lowered further until a maximal population spike was obtained on the second most dorsal contact point. The leads of the scalp-, depth- and vagus electrodes were assembled in a head cap on the skull of the rat using dental cement. Post-operative analgesia was provided by subcutaneously administering buprenorphine $(0.03 \mathrm{mg} / \mathrm{kg})$ and meloxicam ( $1 \mathrm{mg} / \mathrm{kg}$, daily for up to 2 days after surgery). Animals were allowed to recover from surgery during 2-3 weeks. After the experiments, electrode localization was histologically verified on hippocampal slices.

\section{Electrophysiology}

After recovery, rats were housed in dedicated cages for in vivo electrophysiological recording and stimulation via a tethered system. In these cages, EEG signals were recorded via a head stage, carrying unity gain preamplifiers, and an electrical commutator connected to custom-built amplifiers (gain: x100; band-width: $0.13 \mathrm{~Hz}-5.8 \mathrm{kHz}$ ). A stainless-steel epidural electrode placed over the right frontal lobe was used as ground and reference electrode. Signals were high-pass filtered at $0.1 \mathrm{~Hz}$ and amplified 100 times. A data acquisition card (NI-USB-6259, National Instruments, USA, TX) digitizes the EEG (at $1 \mathrm{kHz}$ ) and EP signals (at $20 \mathrm{kHz}$ ) with a 16-bit dynamic range and a $\pm 3.05 \mu \mathrm{V}$ resolution. Signals were stored on a PC for offline analysis. Stimulation pulses were delivered by custom-built constant current stimulators.

To establish the intensity of Schaffer collateral stimulation for recording of CA1 EPs, Schaffer collaterals were stimulated with $100 \mu$ s electrical pulses and intensities ranging from 0 to $440 \mu \mathrm{A}$ with incremental steps of $20 \mu \mathrm{A}$. Per intensity level, 10 electrical pulses were delivered and the average EP was calculated. The current intensity which evoked a PS with $80 \%$ of its maximal amplitude $\left(\mathrm{I}_{80}\right)$ was selected for further experiments in each animal.

Fig.1 gives a schematic overview of the experiment. A first experimental session, termed the VNS session, consisted of a two-hour baseline period, a four-hour rapid cycle VNS period (7s on/18s off, $1 \mathrm{~mA}$, square wave biphasic pulses, $250 \mu \mathrm{s} /$ phase, $30 \mathrm{~Hz}$ ) and a four-hour wash-out period. A second experimental session, termed the VNS+heating session, and conducted at least 3 days after the first, was similar to the VNS session except that external heating was applied to the rat during the third and fourth hour of VNS via an infrared radiation lamp (Philips, 150 W, HP3616/01) placed at a distance of $30 \mathrm{~cm}$ in front of the home cage. 
The VNS and VNS+heating sessions each consisted of 1440 recording cycles of 25 seconds. (Fig.1). During each cycle, ten seconds of EEG was recorded (second 11-21). During VNS periods, 7 seconds of VNS was administered (second 0-7) corresponding to rapid cycle VNS (7s on/ 18s off). Every 20 minutes, 6 EPs were recorded in 6 subsequent cycles (one per cycle at second 9), immediately followed by measuring of core temperature which was acquired rectally.

\section{Electrophysiological analysis}

Three parameters were extracted from the EPs (Fig 2). 1) fEPSP slope by fitting a line from the fEPSP start until the PS onset using the least squares method; 2) PS amplitude by measuring the vertical distance between the PS peak and the line between the positive fEPSP local peaks before and after the PS; 3) PS latency which is the time between the stimulation onset and the PS peak. Per 20 minutes, parameters of EPs $(n=6)$ were averaged.

Three EEG parameters were extracted. 1) normalized theta (4-12 Hz) frequency band power; 2) normalized gamma $(30-100 \mathrm{~Hz})$ frequency band power and 3) theta peak frequency. EEG was filtered off-line with a first order Butterworth high pass filter (cut-off $2 \mathrm{~Hz}$ ) and a notch filter at 50 Hz. To obtain local hippocampal EEG, signals from the electrode contacts with the smallest and largest evoked fEPSP were subtracted. Each 10-second EEG was split into 19 segments of 1s, overlapping by $0.5 \mathrm{~s}$. Using Fast Fourier transform a power spectrum (2-100 Hz, $1 \mathrm{~Hz}$ resolution) was calculated for each 1-second segment. All 19 power spectra were averaged to obtain one power spectrum for each 10-second EEG. Per 20-minutes power spectra of all 48 cycles were averaged and total $(2-100 \mathrm{~Hz})$, theta $(4-12 \mathrm{~Hz})$ and gamma $(30-100 \mathrm{~Hz})$ frequency band power were determined by integrating the power of all $1-\mathrm{Hz}$ frequency bins between respective frequency limits. For each epoch total, theta and gamma band power were normalized by dividing them with the average total power of all epochs in that experimental session. Theta peak frequency was determined as the $1-\mathrm{Hz}$ frequency bin with the highest power within the theta frequency band. EEG segments were rejected if total power of that segment deviated more than 3 standard deviations from the total mean power.

\section{Data analysis and Statistics}

All data were processed using Matlab 2007b (The mathwork Inc., Natick, USA). Statistical analyses were performed using SPSS Statistics 22 (IBM Corporation, Armonk, New York, US) and R 3.3.2 (R Development Core Team). 
To evaluate the effect of VNS and external heating on body temperature and hippocampal EEG/EP, a linear mixed effects model was fitted to the data with session (VNS vs VNS+heating); time [final 20 min baseline; final 20 min of $2 \mathrm{~h}$ VNS; final 20 min of 4h VNS; first 20 min washout; final 20 min washout] and the interaction between session and time as fixed effects. Significant interactions between session and time were found for all parameters. Therefore pairwise comparisons between the 20min-segments are reported per session. Bonferroni correction for post-hoc testing was used. Statistical significance was considered at $p<0.05$. All values are expressed as mean \pm standard error of the mean.

\section{Results}

\section{VNS and temperature effects.}

In the VNS and VNS+heating session body temperature decreased $(\mathrm{p}<0.001)$ from respectively $36.9 \pm 0.4{ }^{\circ} \mathrm{C}$ and $37.2 \pm 0.4{ }^{\circ} \mathrm{C}$ during baseline to respectively $33.5 \pm 0.4^{\circ} \mathrm{C}$ and $32.7 \pm 0.4{ }^{\circ} \mathrm{C}$ after 2 hours of VNS. (Fig.3). In the VNS session, core temperature remained hypothermic though did not further reduce during the last two hours of VNS. After 4 hours of VNS, core temperature was $33.1 \pm 0.4{ }^{\circ} \mathrm{C}$ (Fig.3). During the first 20 minutes of wash-out body temperature was still hypothermic $\left(33.3 \pm 0.4{ }^{\circ} \mathrm{C}\right)$ but then gradually normalized. At the end of the experiment core temperature was $36.8 \pm 0.4^{\circ} \mathrm{C}$.

In the VNS+heating session, rats were exposed to external heating during the last two hours of VNS which increased their body temperature to $36.7 \pm 0.4^{\circ} \mathrm{C}$. This is significantly $(\mathrm{p}<0.001)$ higher than the hypothermic values after two hours of VNS and not significantly different from core temperature at baseline. During the first 20 minutes of washout and after 4 hours of washout body temperature remained at baseline levels.

\section{The effect of VNS on CAl evoked potentials}

In the VNS session, VNS significantly affected fEPSP slope and PS amplitude and latency of CA1 EPs (see Fig. 2 for representative sample). fEPSP slope was $0.63 \pm 0.48 \mathrm{mV} / \mathrm{ms}$ at baseline and was significantly reduced by $0.22 \pm 0.07 \mathrm{mV} / \mathrm{ms}$ ( $\mathrm{p}<0.01$, Fig.4A) after 2 hours of VNS, by $0.19 \pm$ $0.07 \mathrm{mV} / \mathrm{ms}(\mathrm{p}<0.05)$ after 4 hours of VNS and by $0.19 \pm 0.07 \mathrm{mV} / \mathrm{ms}$ in the first 20 minutes of wash-out ( $<<0.05$ ). PS amplitude was $1.00 \pm 0.13 \mathrm{mV}$ at baseline and increased by $0.42 \pm 0.17 \mathrm{mV}$ 
(p>0.05, Fig.4D) after 2 hours of VNS, by $0.66 \pm 0.17 \mathrm{mV}(\mathrm{p}<0.01)$ after 4 hours of VNS and by $0.79 \pm 0.17 \mathrm{mV}(\mathrm{p}<0.001)$ in the first 20 minutes of wash-out. PS latency was $8.56 \pm 0.24 \mathrm{~ms}$ at baseline and significantly increased by $1.19 \pm 0.32 \mathrm{~ms}$ (p<0.01, Fig.4G) after 2 hours of VNS, by $1.41 \pm 0.32 \mathrm{~ms}(\mathrm{p}<0.001)$ after 4 hours of VNS and was still increased by $1.16 \pm 0.32 \mathrm{~ms}(\mathrm{p}<0.01)$ in the first 20 minutes of wash-out. Four hours after switching off VNS, EP parameters were no longer different from baseline.

In the VNS+heating session, 2 hours of VNS had similar effects on EP parameters as in the VNS session. fEPSP slope was $0.58 \pm 0.05 \mathrm{mV} / \mathrm{ms}$ ( $\mathrm{p}>0.05$, Fig4.A) at baseline and was reduced by 0.13 $\pm 0.06 \mathrm{mV} / \mathrm{ms}$ (p>0.05, Fig.4A). PS amplitude was $1.40 \pm 0.12 \mathrm{mV}$ at baseline and increased by $0.39 \pm 0.16 \mathrm{mV}(\mathrm{p}>0.05$, Fig.4D). PS latency was $8.11 \pm 0.24 \mathrm{~ms}$ at baseline and significantly increased by $1.67 \pm 0.31 \mathrm{~ms}$ ( $\mathrm{p}<0.001$, Fig.4G). By the end of the 4 hour VNS period, when external heating had restored core temperature to baseline levels, fEPSP slope increased by $0.07 \pm 0.06$ $\mathrm{mV} / \mathrm{ms}$ to $0.52 \pm 0.45 \mathrm{mV} / \mathrm{ms}$ (p>0.05, Fig4.A). PS amplitude decreased by $1.12 \pm 0.16 \mathrm{mV}$ (p<0.001, Fig.4D) to $0.68 \pm 0.12 \mathrm{mV}$ which is significantly lower compared to baseline and after 2 hours of VNS. PS latency decreased by $1.26 \pm 0.31 \mathrm{~ms}$ to $8.52 \pm 0.22 \mathrm{~ms}$ which is not significantly different from baseline (Fig.G). During the first 20 minutes of washout, PS amplitude significantly increased by $0.68 \pm 0.16 \mathrm{mV}$ ( $\mathrm{p}<0.01$, Fig.4D) back to baseline levels $(1.36 \pm 0.12 \mathrm{mV}$ ), while fEPSP slope and PS latency remained at baseline levels $(0.55 \pm 0.04 \mathrm{mV} / \mathrm{ms}$, Fig.4A and $8.39 \pm$ $0.21 \mathrm{~ms}$, Fig.G respectively).

\section{The effect of VNS on CAI EEG}

In the VNS session, VNS significantly affected total $(2-100 \mathrm{~Hz})$ EEG power, normalized theta and gamma band power and theta peak frequency. Total power was reduced by $21.0 \pm 2.0 \%$ (Fig.1, $\mathrm{p}<0.001)$ after 2 hours of VNS compared to baseline levels, by $18.0 \pm 2.0 \%(\mathrm{p}<0.001)$ after 4 hours of VNS and by $16.3 \pm 2.0 \%(\mathrm{p}<0.001)$ in the first 20 minutes of the washout period. Normalized theta and gamma band power were reduced by $9.9 \pm 0.9 \%(\mathrm{p}<0.001$, Fig.5D) and $5.0 \pm 0.6 \%$ $(\mathrm{p}<0.001$, Fig.5G) after 2 hours of VNS compared to baseline levels, by $9.0 \pm 0.9 \%(\mathrm{p}<0.001)$ and $4.7 \pm 0.6 \%(\mathrm{p}<0.001)$ after 4 hours of VNS and by $7.3 \pm 0.9 \%(\mathrm{p}<0.001)$ and $4.1 \pm 0.6 \%(\mathrm{p}<0.001)$ during the first 20 minutes of washout respectively. Theta peak frequency was $5.97 \pm 0.85 \mathrm{~Hz}$ at baseline and was reduced by $0.88 \pm 0.12 \mathrm{~Hz}(\mathrm{p}<0.001$, Fig.J) after 2 hours of VNS compared to baseline, by $1.02 \pm 0.12 \mathrm{~Hz}(\mathrm{p}<0.001)$ after 4 hours of $\mathrm{VNS}$ and by $0.72 \pm 0.12 \mathrm{~Hz}(\mathrm{p}<0.001)$ during 
the first 20 minutes of washout. At the end of the 4-hour washout period, all EEG parameters returned to baseline levels.

In the VNS+heating session, 2 hours of VNS had similar effects on EEG parameters as in the VNS session. Total power was reduced by $21.2 \pm 2.0 \%$ (p<0.001, Fig.5A). Normalized theta and gamma band power were reduced by $9.0 \pm 0.9 \%$ (p<0.001, Fig5.D) and $4.5 \pm 0.6 \%$ ( $<<0.001$, Fig5.G). Theta peak frequency was reduced by $1.15 \pm 0.12 \mathrm{~Hz}$ compared to baseline $(6.06 \pm 0.08 \mathrm{~Hz}$, $\mathrm{p}<0.001$, Fig.5J).

When rats were exposed to external heating after 2 hours of VNS and their temperature was normalized by the end of the $4 \mathrm{~h}$ VNS period, total EEG power, normalized theta and gamma band power and theta peak frequency significantly increased by $16.1 \pm 2.1 \%(\mathrm{p}<0.001), 3.2 \pm 0.9 \%$ $(\mathrm{p}<0.01), 3.3 \pm 0.6 \%(\mathrm{p}<0.001)$ and $0.74 \pm 0.12(\mathrm{p}<0.001) \mathrm{Hz}$ respectively. Normalized theta power and theta peak frequency were still significantly different from baseline $(\mathrm{p}<0.01$ and $\mathrm{p}<0.001)$, while normalized gamma power was not. During the first 20 minutes washout normalized theta power was significantly increased by $6.0 \pm 0.9 \%(\mathrm{p}<0.001)$ and back to baseline levels, while theta peak frequency $(5.7 \pm 0.08 \mathrm{~Hz})$ remained significantly lower than baseline. Normalized gamma band power and total EEG power remained at baseline levels. At the end of the 4 hour washout period, none of the EEG parameters differed from baseline.

\section{Discussion}

This study demonstrates that rapid cycle VNS modulates EPs and EEG recorded from hippocampal CA1. EP changes reflect decreased synaptic efficacy (decreased fEPSP slope and increased PS latency) but at the same time increased neuronal excitability (increased PS amplitude). EEG changes reflect reduced spontaneous activity (decreased total power, normalized theta and gamma band power) and slowing of oscillatory patterns (reduced theta peak frequency). In previous studies we found identical electrophysiological effects of VNS in the dentate gyrus $(10,11)$. This study also confirmed our previous finding that 2 hours of rapid cycle VNS induces a decrease in body temperature of $3^{\circ} \mathrm{C}$. Doubling the duration of rapid cycle VNS did not further decrease body temperature indicating maximal hypothermia after 2 hours of rapid cycle VNS in standard lab conditions. We hypothesize that VNS-induced hypothermia is caused by modulation of vagal nerve fibers sending temperature information from cold and warm receptors via NTS glutamatergic neurons to the external and dorsal lateral parabrachial nucleus (LPB) of the brainstem respectively 
(12). The external and dorsal LPB activate respectively GABAergic and glutamatergic relay neurons both controlling the activity of warm-sensitive neurons of the medial preoptic nucleus (PO) of the hypothalamus (13-15). In case firing rate of PO warm-sensitive neurons increases, peripheral mechanisms are activated for reduction of body temperature (16). VNS might preferentially activate dorsal LPB neurons which in turn activate warm-sensitive PO neurons resulting in active cooling and hypothermia. cFos activation studies in rats showed that acute VNS among other brain regions activates LPB $(17,18)$. Unfortunately, no distinction was made between different LPB subnuclei.

VNS-induced hypothermia in rats is mediated by vasodilatation in tail and paws and release of heat to the environment via the circulation (8). In this study ambient temperature was increased using an infrared lamp to normalize body temperature during VNS. As a result VNS effects on fEPSP slope, PS latency are almost completely negated indicating that decreased synaptic efficacy is closely associated with VNS-induced hypothermia. The effects of VNS on total power, gamma and theta band power and theta peak frequency were also significantly reduced, indicating that reduction in spontaneous neuronal activity and slowing of theta pattern are at least partially related to VNS-induced hypothermia.

The association between VNS-induced hypothermia and changes in hippocampal electrophysiology are in line with previous in vitro (19) and in vivo (20-22) evidence.

Experiments on hippocampal slices have shown that both amplitude and duration of evoked action potentials of CA1 pyramidal cells are increased at low temperatures, resulting in increased population spike amplitudes (23). In vivo experiments showed decreased fEPSP slope and increased PS latency/amplitude of DG EPs when rats were cooled while exposing rats to external heating had opposite effects(20-22). The appearance of hippocampal theta rhythm in rats is also temperature dependent. Theta oscillations appear in the hippocampus at core temperatures ranging from $23^{\circ}$ to $42^{\circ} \mathrm{C}$, with frequencies ranging from 2 to $12 \mathrm{~Hz}(24)$. Theta peak frequency decreases linearly with decreasing temperature, while its amplitude only decreases below $30^{\circ} \mathrm{C}(24,25)$.

This study also provided arguments for temperature-independent effects of VNS on hippocampal electrophysiology. In normothermic rats PS, amplitude, theta band power, and theta peak frequency are significantly reduced compared to baseline during VNS. Switching off VNS resulted in a significant and complete normalization of these parameters. Reversal of VNS effects on PS 
amplitude upon normalizing body temperature indicates that hypothermia can mask a reduction in neuronal excitability in response to VNS.

In epileptic tissue, EPs are characterized by a lower latency and higher amplitude (26). The VNSinduced effect on the PS amplitude in normothermic rats may be related to its anti-epileptic mechanism. In normothermic rats PS latency seems not affected by VNS, indicating that the slowing of conduction is for the most part caused by VNS-induced hypothermia. This suggests that, without hypothermia as a confounder, the effect of VNS on excitability is most likely a postsynaptic phenomenon.

Taken together, our data show that rapid cycle VNS-induced hypothermia is most likely responsible for reduced synaptic efficacy in the hippocampus (evidenced by a reduced slope and increased PS latency) and reduction of power of EEG in gamma frequency band. VNS has the potential to decrease the excitability of CA1 neurons (reduced PS amplitude) but this effect may be masked by the VNS-induced hypothermia. Changes in theta oscillations in the CA1 during VNS are caused by hypothermic and non-hypothermic effects of VNS.

A caveat of this study is that true causality could not be established between the hypothermic state and hippocampal electrophysiological changes, as the normalization of the body temperature was realized by interfering with the peripheral effectors (increasing ambient temperature rendering the induced vasodilation ineffective) rather than interfering with the central thermoregulatory system (i.e. hypothalamic manipulation). Also, as no heating without VNS was studied, any residual effects on the electrophysiological parameters inherent to VNS similar to and therefore masked by heating cannot be excluded.

In general, our study points out that the hypothermic effects of VNS in rats is a potential confounder that needs to be taken into account when studying in vivo effects of VNS on brain excitability and beyond in rats. Experiments screening for VNS parameters in rats that are not associated with hypothermia are ongoing at the time of writing. Translation of preclinical VNS results in rodents to patients requires caution.

\section{Acknowledgements}


Wouter Van Lysebettens is supported by a stategic basic research grant provided by Flemish Research Fund (FWO, grantnr. 141294). Prof. Dr. K. Vonck and Prof. Dr. R. Raedt are supported by the Special Research Funds (BOF) of Ghent University. Prof. Dr. P. Boon is supported by research grants from FWO, BOF and Ghent University Hospital.

\section{References}

1. Ben-Menachem E. Vagus-nerve stimulation for the treatment of epilepsy. Lancet Neurol. 2002;1(8):477-82.

2. Raedt R, Clinckers R, Mollet L, Vonck K, El Tahry R, Wyckhuys T, et al. Increased hippocampal noradrenaline is a biomarker for efficacy of vagus nerve stimulation in a limbic seizure model. J Neurochem. 2011;117(3):461-9.

3. Schwarz LA, Luo L. Organization of the locus coeruleus-norepinephrine system. Curr Biol. 2015;25(21):R1051-R6.

4. Woodbury DM, Woodbury JW. Effects of vagal stimulation on experimentally induced seizures in rats. Epilepsia. 1990;31 Suppl 2:S7-19.

5. Larsen LE, Wadman WJ, Marinazzo D, van Mierlo P, Delbeke J, Daelemans S, et al. Vagus Nerve Stimulation Applied with a Rapid Cycle Has More Profound Influence on Hippocampal Electrophysiology Than a Standard Cycle. Neurotherapeutics. 2016;13(3):592-602.

6. Shen H, Fuchino Y, Miyamoto D, Nomura H, Matsuki N. Vagus nerve stimulation enhances perforant path-CA3 synaptic transmission via the activation of beta-adrenergic receptors and the locus coeruleus. Int J Neuropsychopharmacol. 2012;15(4):523-30.

7. Ura H, Sugaya Y, Ohata H, Takumi I, Sadamoto K, Shibasaki T, et al. Vagus nerve stimulation induced long-lasting enhancement of synaptic transmission and decreased granule cell discharge in the hippocampal dentate gyrus of urethane-anesthetized rats. Brain Res. 2013;1492:63-71.

8. Larsen LE, Lysebettens WV, Germonpre C, Carrette S, Daelemans S, Sprengers M, et al. Clinical Vagus Nerve Stimulation Paradigms Induce Pronounced Brain and Body Hypothermia in Rats. Int J Neural Syst. 2017;27(5):1750016.

9. El Tahry R, Mollet L, Raedt R, Delbeke J, De Herdt V, Wyckhuys T, et al. Repeated assessment of larynx compound muscle action potentials using a self-sizing cuff electrode around the vagus nerve in experimental rats. J Neurosci Methods. 2011;198(2):287-93.

10. Larsen LE, Wadman WJ, Marinazzo D, van Mierlo P, Delbeke J, Daelemans S, et al. Vagus Nerve Stimulation Applied with a Rapid Cycle Has More Profound Influence on Hippocampal Electrophysiology Than a Standard Cycle. Neurotherapeutics. 2016.

11. Larsen LE, Wadman WJ, van Mierlo P, Delbeke J, Grimonprez A, Van Nieuwenhuyse B, et al. Modulation of Hippocampal Activity by Vagus Nerve Stimulation in Freely Moving Rats. Brain Stimul. 2016;9(1):124-32.

12. Saper CB. The central autonomic nervous system: conscious visceral perception and autonomic pattern generation. Annu Rev Neurosci. 2002;25:433-69.

13. Morrison SF, Nakamura K. Central neural pathways for thermoregulation. Front Biosci (Landmark Ed). 2011;16:74-104.

14. Nakamura K, Morrison SF. A thermosensory pathway that controls body temperature. Nat Neurosci. 2008;11(1):62-71. 
15. Nakamura K, Morrison SF. A thermosensory pathway mediating heat-defense responses. Proc Natl Acad Sci U S A. 2010;107(19):8848-53.

16. Morrison SF. Central control of body temperature. F1000Res. 2016;5.

17. Cunningham JT, Mifflin SW, Gould GG, Frazer A. Induction of c-Fos and DeltaFosB

immunoreactivity in rat brain by Vagal nerve stimulation. Neuropsychopharmacology : official publication of the American College of Neuropsychopharmacology. 2008;33(8):1884-95.

18. Saleh TM, Cechetto DF. Peptide changes in the parabrachial nucleus following cervical vagal stimulation. J Comp Neurol. 1996;366(3):390-405.

19. Thompson SM, Masukawa LM, Prince DA. Temperature dependence of intrinsic membrane properties and synaptic potentials in hippocampal CA1 neurons in vitro. J Neurosci. 1985;5(3):817-24.

20. Cain DP, Hargreaves EL, Boon F. Brain temperature- and behavior-related changes in the dentate gyrus field potential during sleep, cold water immersion, radiant heating, and urethane anesthesia. Brain Res. 1994;658(1-2):135-44.

21. Moser E, Mathiesen I, Andersen P. Association between brain temperature and dentate field potentials in exploring and swimming rats. Science. 1993;259(5099):1324-6.

22. Moser El, Andersen P. Conserved spatial learning in cooled rats in spite of slowing of dentate field potentials. J Neurosci. 1994;14(7):4458-66.

23. Andersen $\mathrm{P}$, Moser El. Brain temperature and hippocampal function. Hippocampus. 1995;5(6):491-8.

24. Whishaw IQ, Vanderwolf $\mathrm{CH}$. Hippocampal EEG and behavior: effects of variation in body temperature and relation of EEG to vibrissae movement, swimming and shivering. Physiol Behav. 1971;6(4):391-7.

25. Kowalczyk T, Golebiewski H, Eckersdorf B, Konopacki J. Window effect of temperature on carbachol-induced theta-like activity recorded in hippocampal formation in vitro. Brain Res. 2001;901(12):184-94.

26. Queiroz CM, Gorter JA, Lopes da Silva FH, Wadman WJ. Dynamics of evoked local field potentials in the hippocampus of epileptic rats with spontaneous seizures. J Neurophysiol. 2009;101(3):1588-97.

\section{Figures}

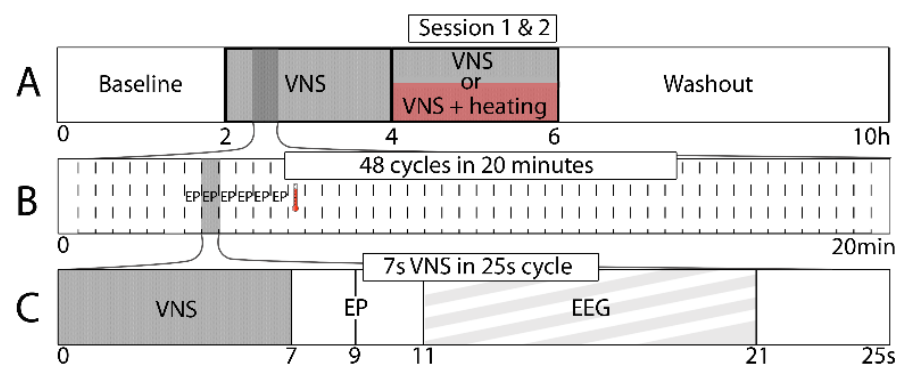

Figure 1: Schematic illustration of recording and stimulation protocol. A. In experimental session 1, two hours of baseline was followed by four hours of VNS and four hours washout. Experimental session 2 is similar to experimental session 1 except for application of infrared heating in combination with VNS in the third and fourth hour of VNS. B. Each 20 minute period consists of 48 cycles lasting 25 seconds. In 6 of these cycles a hippocampal evoked potential is recorded, followed by core temperature registration. C. In each cycle 10 seconds of EEG is recorded from second 11 
to 21. In 6/48 cycles an EP is registered at the ninth second. In the VNS hours, VNS is administered in the first seven seconds of each cycle.

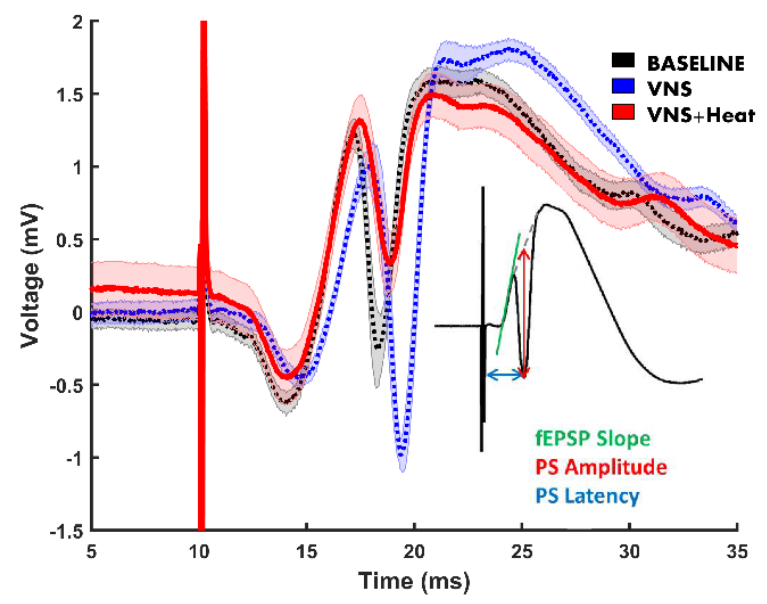

Figure 2: Representative sample of an evoked potential during baseline (black), VNS (blue) and VNS with external heating (red). Shaded areas represent the $95 \%$

CI. A schematic representation of the outcome parameters, field excitatory post synaptic potential (fEPSP) slope, population spike amplitude, and latency are depicted in small in the lower right corner. 
A The Effect of VNS on rectal temperature

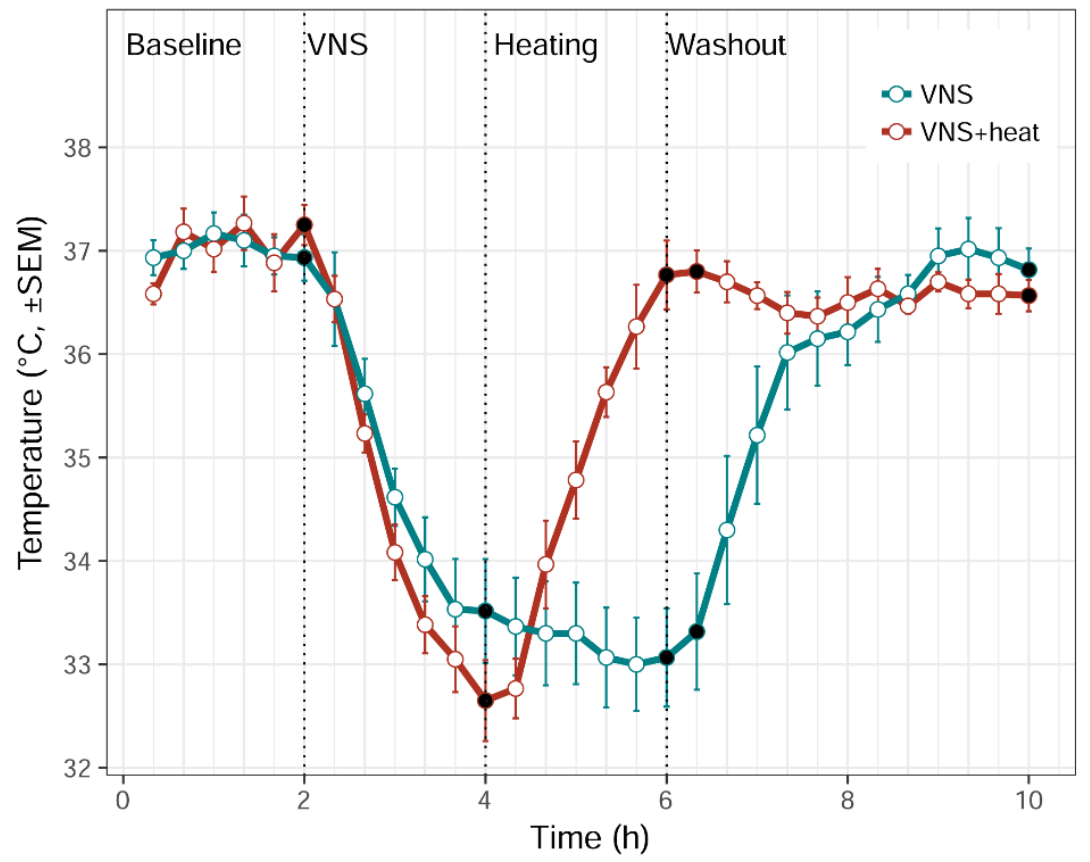

Figure 3: A) Core temperature of rats during VNS (blue) or VNS+heating session (red). 20-minute periods indicated by black dots were statistically compared. 
A

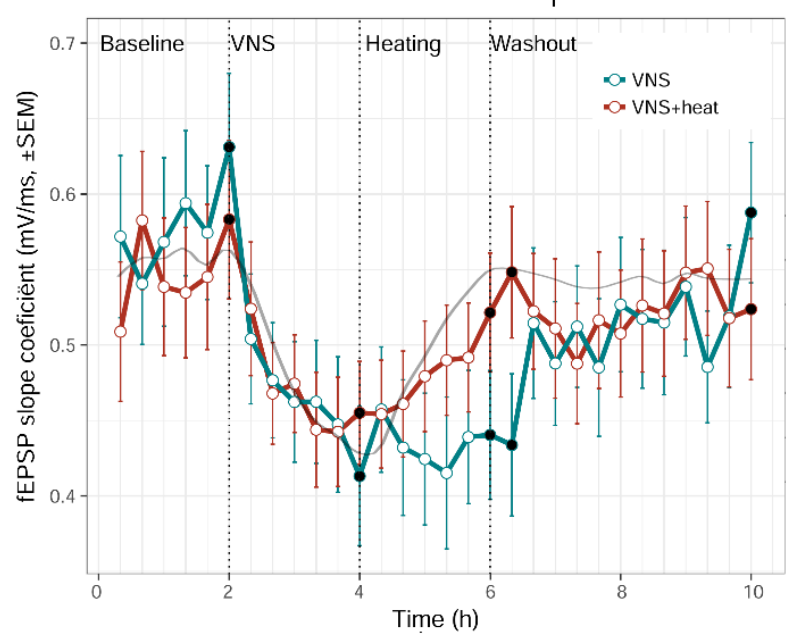

D The Effect of VNS on CA1 population spike amplitude

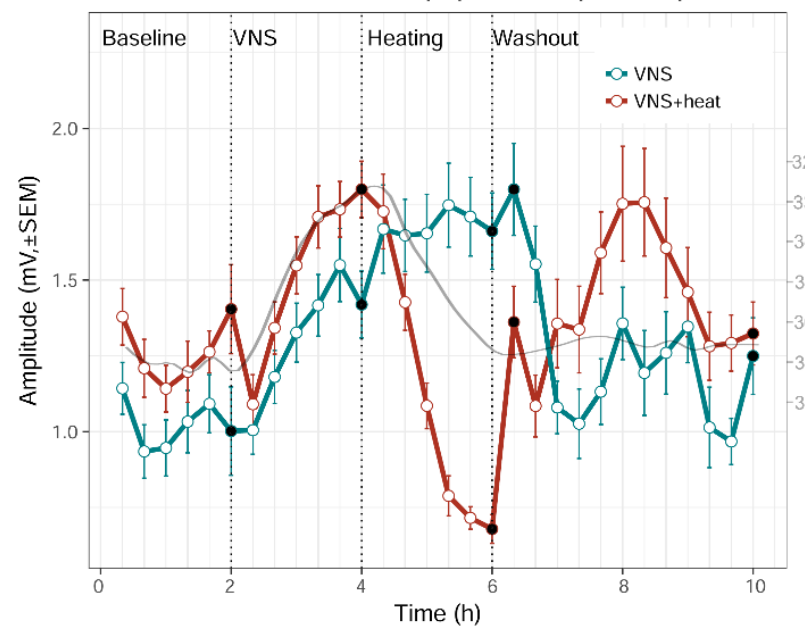

G The Effect of VNS on CA1 population spike latency

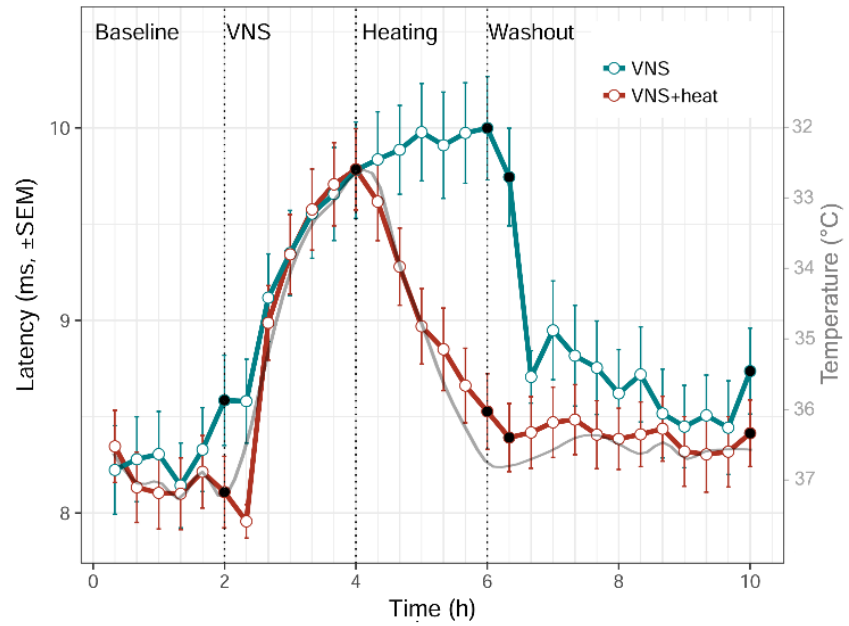

B
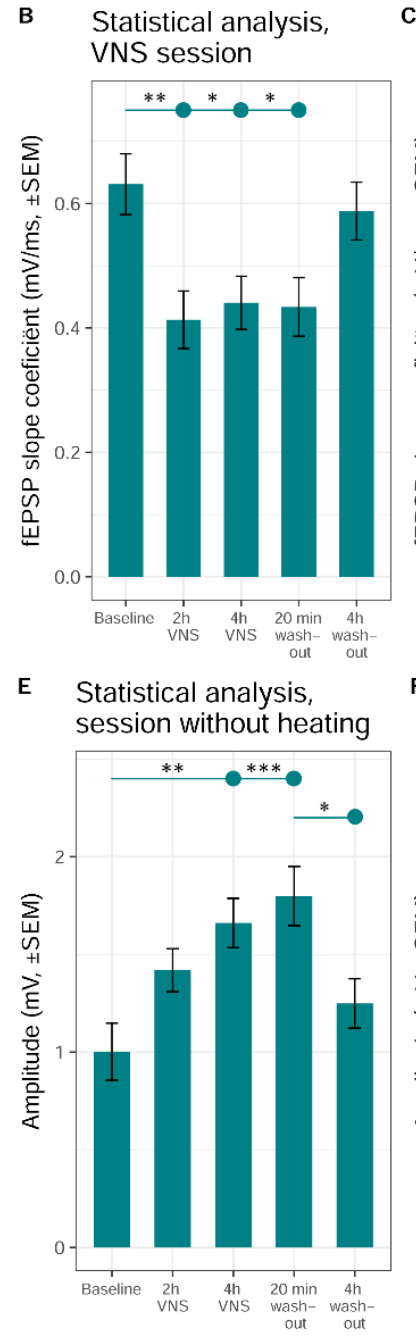

H

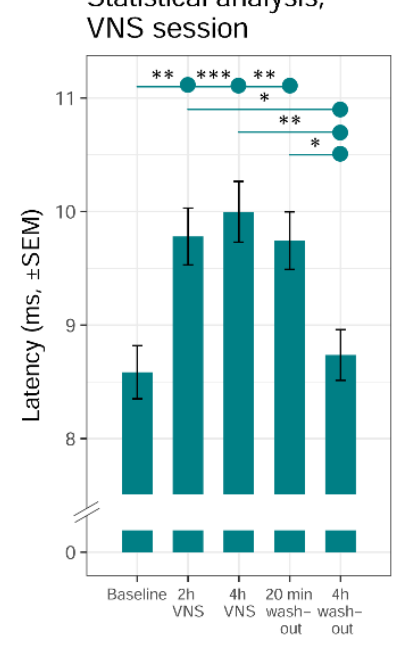

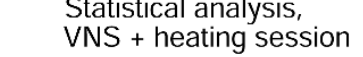

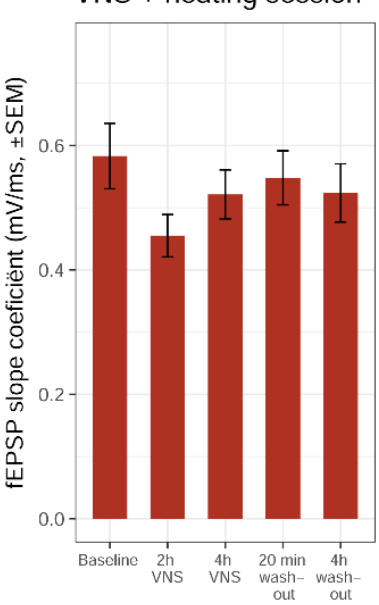

F Statistical analysis, session with heating

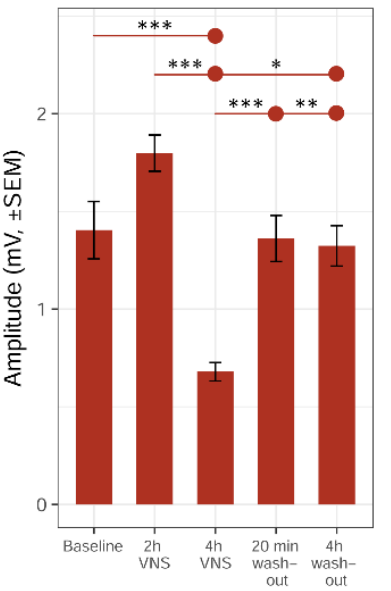

I Statistical analysis, VNS + heating session

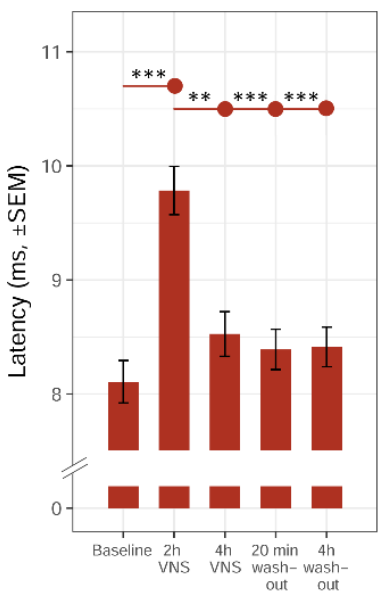

Figure 4: Graphic representation of VNS-induced effects on EP outcome parameters. Changes in PS latency (A), PS amplitude (D) and fEPSP slope (G) of CA1 EPs during the VNS and VNS+heating sessions. Core temperature measured during the VNS+heating session is plotted in grey with axis at right side (note that the temperature y-axis in A and D is inversed compared to G). In the VNS session, PS amplitude and latency are increased, while fEPSP slope is decreased. During VNS+heating, PS latency and amplitude decrease while fEPSP slope increases. 20-minute periods indicated by black dots were statistically compared. B/C, E/F, G/H. For clarity the y-axes in figures 4B and $4 \mathrm{C}$ were broken. $(\mathrm{p}<0.05=" * ", \mathrm{p}<0.01=" * * ", \mathrm{p}<0.001=" * * * ")$ 
A

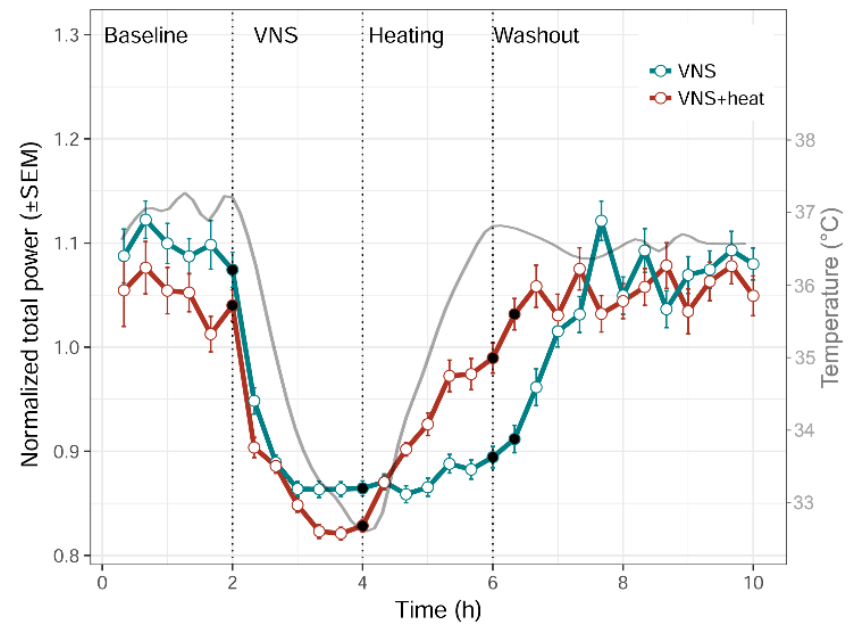

D

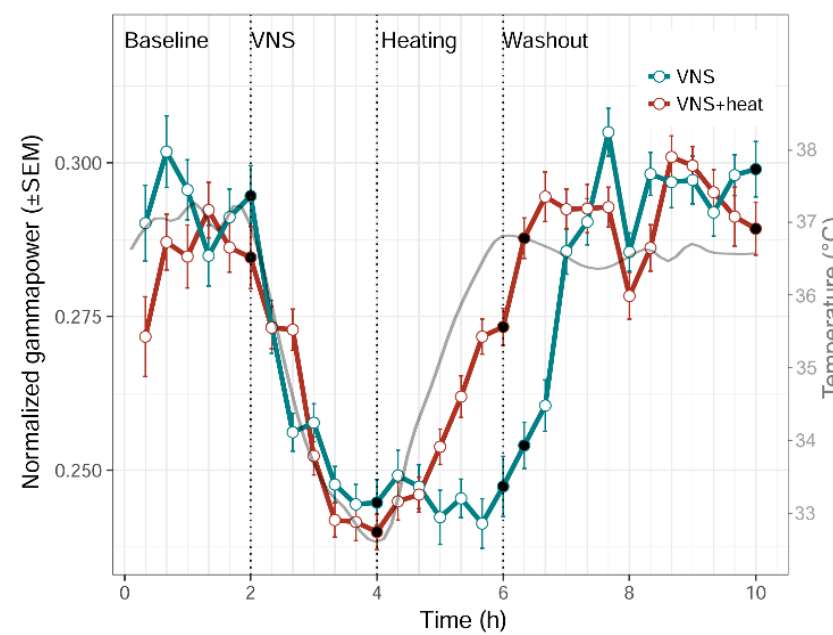

G

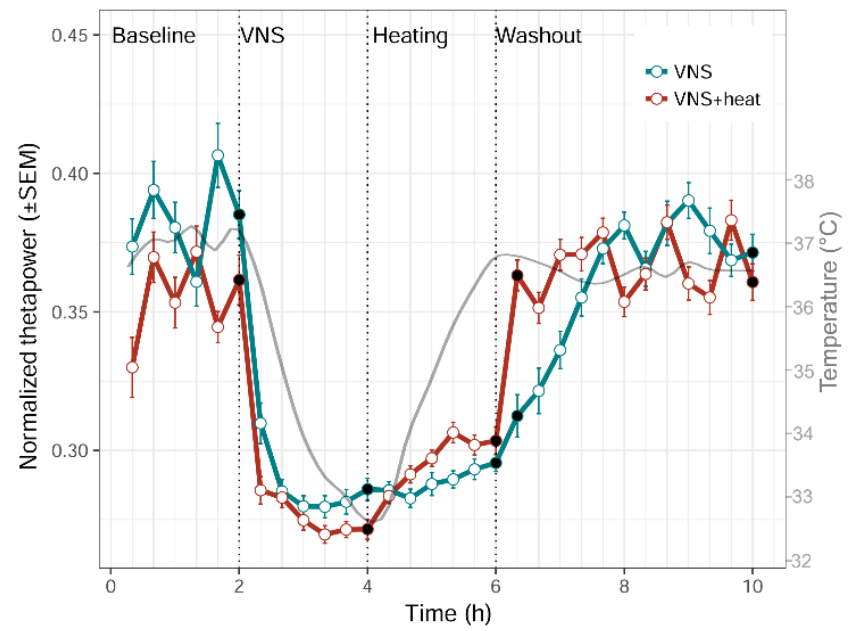

B Statistical analysis, VNS session

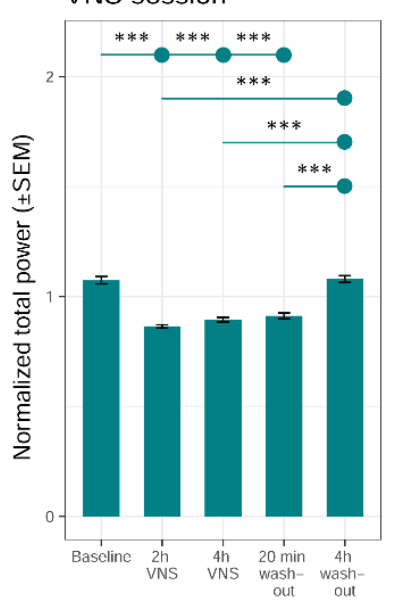

c Statistical analysis,

VNS + heating session

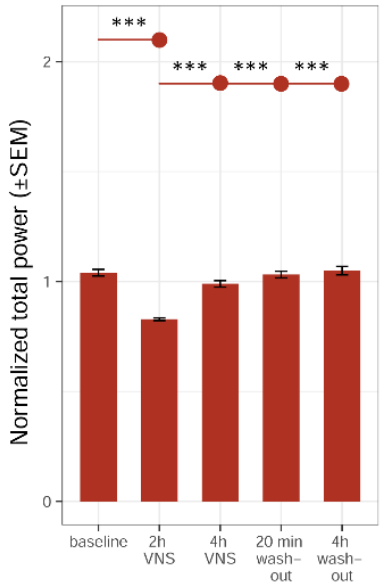

E

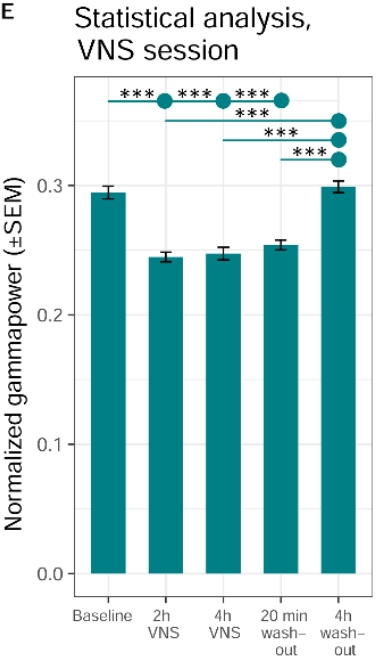

H

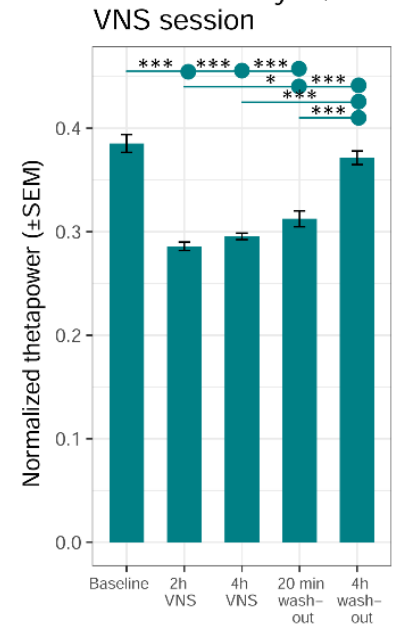

F Statistical analysis,

VNS + heating session

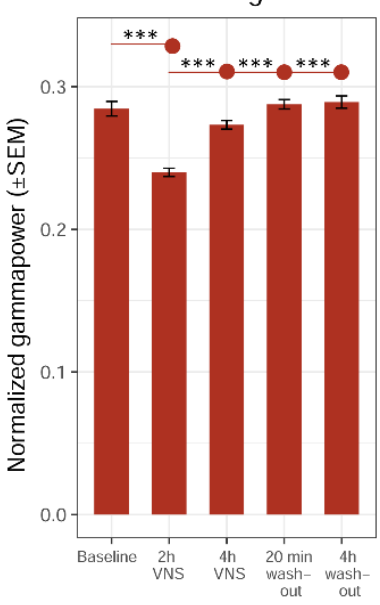

Statistical analysis, VNS + heating session

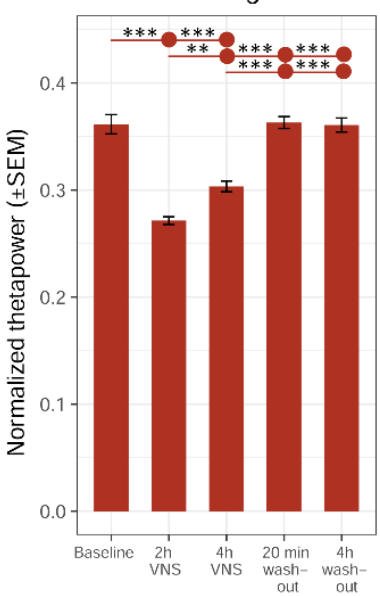



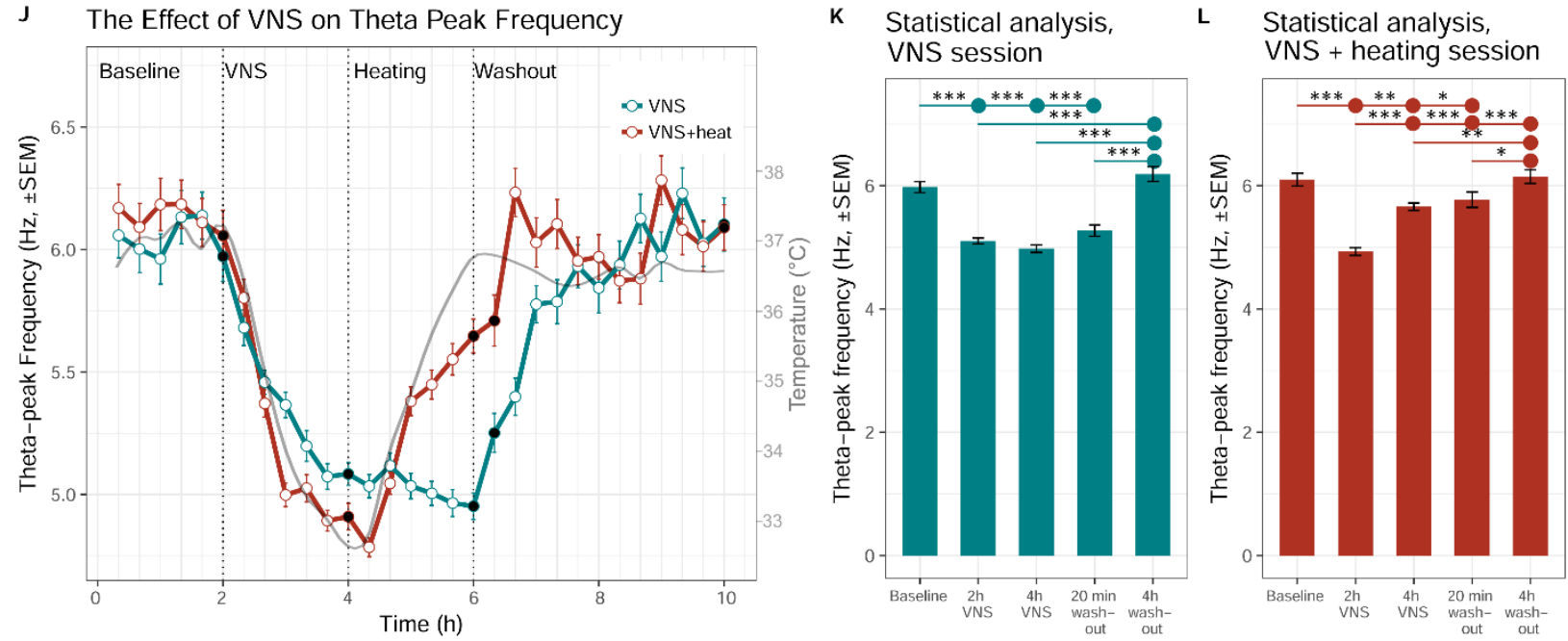

Figure 5: Effect of VNS and VNS+heating on CA1 EEG parameters. Changes in normalized total EEG power (A, 2$100 \mathrm{~Hz})$. Changes in normalized gamma power $(\mathrm{D}, 30-100 \mathrm{~Hz})$, normalized theta power $(\mathrm{G}, 4-12 \mathrm{~Hz})$ and theta peak frequency $(\mathrm{J})$. Theta and gamma power were normalized to the mean total power of the entire session. All parameters are reduced during VNS. During VNS+Heating, normalized gamma power returns to baseline level, theta peak frequency follows the same trend while remaining significantly different from baseline. Theta power experiences a slight increase, and fully returns to baseline once VNS is switched off. 20-minute periods indicated by black dots were statistically compared. (G) Core temperature measured during the VNS+heating session is plotted in grey with axis at right. B/C, E/F,G/H, K/L. (p<0.05="*", p<0.01="**”, p <0.001="***”) 